Virginia Commonwealth University VCU Scholars Compass

2014

\title{
Surface diffusion driven morphological instability in free-standing nickel nanorod arrays
}

Ebtihaj Alrashid Jr.

Virginia Commonwealth University

Dexian Ye

Virginia Commonwealth University, dye2@vcu.edu

Follow this and additional works at: http://scholarscompass.vcu.edu/phys_pubs

Part of the Physics Commons

Alrashid, E., and Ye, D. Surface diffusion driven morphological instability in free-standing nickel nanorod arrays. Journal of Applied Physics, 116, 043501 (2014). Copyright (C) 2014 AIP Publishing LLC.

\section{Downloaded from}

http://scholarscompass.vcu.edu/phys_pubs/139

This Article is brought to you for free and open access by the Dept. of Physics at VCU Scholars Compass. It has been accepted for inclusion in Physics Publications by an authorized administrator of VCU Scholars Compass. For more information, please contact libcompass@vcu.edu. 


\title{
Surface diffusion driven morphological instability in free-standing nickel nanorod arrays
}

\author{
Ebtihaj Alrashid and Dexian Ye \\ Department of Physics, Virginia Commonwealth University, PO Box 842000, Richmond, \\ Virginia 23284-2000, USA
}

(Received 26 March 2014; accepted 11 June 2014; published online 22 July 2014)

\begin{abstract}
Metallic nanostructures are thermodynamically unstable due to the excess of energy of large numbers of surface atoms. Morphological instability, such as Rayleigh breakup, sintering, and coalescence, can be observed at a temperature much lower than the bulk melting point of the metal. We study the morphological and crystalline evolution of well-aligned free-standing nickel nanorod arrays at elevated temperatures up to $600^{\circ} \mathrm{C}$. The as-deposited nickel nanorods are faceted with sharp nanotips, which are deformed at annealing temperatures higher than $400{ }^{\circ} \mathrm{C}$ due to strong surface diffusion. A mud-crack like pattern is formed in the samples annealed above $400{ }^{\circ} \mathrm{C}$, leading to the generation of interconnected porous structure. Meanwhile, the X-ray diffraction reveals the recrystallization of nickel nanocrystals when annealed from 300 to $600{ }^{\circ} \mathrm{C}$. (C) 2014 AIP Publishing LLC. [http://dx.doi.org/10.1063/1.4884878]
\end{abstract}

\section{INTRODUCTION}

Metallic nanostructures in the forms of nanoparticle, nanotube, nanorod, nanowire, and nanospring display unique physical and chemical properties due to the geometry and confinement effects at the nanometer scales. ${ }^{1}$ In metallic nanostructures, atoms on the surface have unsaturated bonds and hence become more active and mobile than in the bulk. ${ }^{1-3}$ The enhanced chemical activity of metallic nanostructures has been exploited to improve the efficiency and selectivity of several important catalytic reactions. ${ }^{3-6}$ The efficiency and selectivity of metallic nanocatalysts strongly depend on the material properties, as well as the size, shape, and the arrangement of atomic planes on the surface of the nanostructures. ${ }^{5}$ Thus, it is ideal to maintain the geometrical and crystalline structures of those nanocatalysts in chemical reactions. On the other hand, the mobility of surface atoms can modify the morphological and crystallographic structures at a moderate temperature, causing the instability of metallic nanostructures. ${ }^{7-9}$

Melting-like morphological change has been reported on a variety of metallic nanorods and nanowires when they are subject to thermal or electromagnetic energy stimuli. ${ }^{10-19}$ Individual nanorods and short nanowires are transformed into spherical nanoparticles driven by the surface diffusion, while individual long nanowires can break into a series of discrete nanoparticles with characteristic diameters and spacing of the particles by diffusion. ${ }^{12,15,20-22}$ For example, individual copper nanowires with a diameter of $300 \mathrm{~nm}$ can fragment into nanoparticles at $700{ }^{\circ} \mathrm{C} ;{ }^{13}$ a gold nanowire with a diameter of $87 \mathrm{~nm}$ can break up at $700{ }^{\circ} \mathrm{C} ;{ }^{20}$ and the fragmentation temperature is $900{ }^{\circ} \mathrm{C}$ for nickel nanowires in a diameter of $30 \mathrm{~nm} .{ }^{18}$ The characteristic diameter and the adjacent separation of the nanoparticles after fragmentation are theoretically predicted based on the surface curvature determined diffusion model. ${ }^{23}$ The diameter of the fragments $D$ and the initial diameter of the nanowire $D_{0}$ can be related as $D=3.78 D_{0}$, according to the model established by
Nichols and Mullins. ${ }^{23}$ The prediction has been experimentally verified in the case of copper nanowires supported on a $\mathrm{SiO}_{2}$ substrate, ${ }^{15}$ whereas deviations of the experimental measurements from the theoretical values are also reported. ${ }^{12,20}$ It is necessary to point out that the temperatures required for the morphological transition in metallic nanostructures are much lower than the bulk melting points of the constituent metals. Although the melting point of a metal nanoparticle is lower than the bulk melting point, the deduction of the melting point is just within a few percent of the bulk melting points, especially for the nanostructures with a radius larger than $10 \mathrm{~nm} .{ }^{18,23,24}$ Therefore, the melting of nanowires is not expected at the temperature that causes dramatic morphological transformation. For arrays of nanorods or nanowires, the fragmentation may not be observed if the spacing between the adjacent nanowires is less than a critical distance. In this case, coalescence and fusion of neighboring nanowires may take place before the break up of individual nanowires.

The morphological instability driven by surface diffusion depends on the geometry (particularly the diameter and the aspect ratio of the nanostructures), crystalline structures, and the supporting media of the metallic nanostructures. $^{13,20,25-29}$ It is found in experiments that nanowires supported on a substrate require higher temperature and longer time to break up than free standing ones. ${ }^{25}$ Recently, free standing copper nanorod arrays prepared by the oblique angle deposition technique can fuse together and form a continuous film after annealing at $550{ }^{\circ} \mathrm{C}$ in vacuum. ${ }^{17}$ The temperature of the transition from porous nanorod arrays to dense film is size dependent. It is discovered recently that the transition temperature of copper nanorod arrays can be lowered to $300{ }^{\circ} \mathrm{C}$ if the average diameter of the copper nanorods is reduced from about $100 \mathrm{~nm}$ to $50 \mathrm{~nm} .{ }^{16}$ Oblique angle deposition technique is used to grow the copper nanorod arrays, where the substrate is mounted to a position to receive deposition flux from the off-normal direction. Therefore, the nanorods fabricated by the oblique angle 
deposition method are tilted towards the deposition source and the separation between nanorods is comparable with the diameter of the nanorods $D_{0}$. Thus, the fragmentation of copper nanorods may be suppressed due to this small spacing between the neighboring tilted nanorods. The fundamental question is how the morphology changes in vertically aligned metallic nanorod arrays at elevated temperatures.

Here, we fabricate well aligned nickel nanorod arrays on silicon substrates for the study of morphological and crystallographic evolutions driven by surface diffusion. The nanorods are grown by using the glancing angle deposition technique, which is similar to the oblique angle deposition except the substrate is rotated constantly. The as-prepared nanorod is faceted with a characteristic sharp tip. The tips can sustain the thermal treatment up to $300^{\circ} \mathrm{C}$ but are deformed during the annealing of the samples in vacuum at a temperature higher than $300^{\circ} \mathrm{C}$. A complex morphology is formed after the connection of multiple nanorods at a temperature high enough to initiate the breakdown of the tips. Consequently, a mud-crack like pattern is self-organized and a porous metallic film is resulted after thermal treatment at 500 and $600{ }^{\circ} \mathrm{C}$. The porous metallic film may have potential applications in catalysis, chemical sensing, battery, and fuel cell.

\section{EXPERIMENTAL DETAILS}

Nickel nanorod arrays are fabricated on native oxide covered silicon (100) substrates in a high vacuum DC magnetron sputtering system, which is described in details elsewhere. ${ }^{30}$ In our experiments, the standard RCA cleaning procedures is used to clean the substrates. The glancing angle deposition method is applied to create well-separated, well-aligned nanorod arrays on the substrates. ${ }^{30-32}$ The angle between the central line of the target and the substrate normal is $85^{\circ}$, which is defined as the deposition angle. The center to center distance between the target and the substrate is about $15 \mathrm{~cm}$. When the background pressure drops below $3 \times 10^{-8}$ Torr, the deposition starts with an argon pressure of 3.9 mTorr and $100 \mathrm{~W}$ sputtering power. The heights of nickel nanorod arrays are controlled by the deposition time, which is set to 30,50 , and $90 \mathrm{~min}$. The substrate is continuously rotated by the stepper motor at a speed of 0.5 revolutions per second during deposition. All the samples of nickel nanorod arrays are imaged by a field emission scanning electron microscope (SEM) (Hitachi SU-70) to study the morphology of the samples. Furthermore, the X-ray diffractometer (PANalytical MPD X'Pert pro) is used to analyze the change of crystalline structures of the nickel nanorod arrays before and after the annealing process.

Annealing of nickel nanorod arrays is performed in a split-top tube furnace (Lindbergh/Blue M) at 300, 400, 500, and $600{ }^{\circ} \mathrm{C}$ for $30 \mathrm{~min}$ under vacuum. The processing pressure in the quartz tube is maintained at $\sim 200$ mTorr using a mechanical pump. A continuous flow of premixed forming gas consisting of 5\% hydrogen and 95\% argon gases (ultrahigh-purity grade) is used to protect the samples from oxidation during thermal treatment. The flow of the gases is adjusted to $10 \mathrm{sccm}$ using a mass flow controller (MKS Instruments, 1179 A Mass-flo) during the process.

\section{RESULTS AND DISCUSSION}

In the glancing angle deposition mode of physical vapor deposition, shadowing effect and limited surface diffusion are the two dominant competing mechanisms for the formation of the nanorod arrays. ${ }^{33,34}$ Due to the shadowing effect at deposition angles close to $90^{\circ}$, incident atoms can only reach the very top part of the growing surface. The growth in the area near the valley of the growing surface is terminated due to the blocking of incident atoms, thereby creating wellseparated nanorods at large deposition angles. In glancing angle deposition, the spacing between nearby nanorods is deposition angle dependent. The nanorods are aligned spontaneously along the normal direction of the substrate when the substrate is rotated continuously at a constant rotation speed. Meanwhile, surface diffusion drives the newly deposited atoms to an energetic favorable site to settle down. At near room temperature, only metastable equilibrium of the deposition can be reached by limited surface diffusion. As a result, individual nickel nanorod grown by our technique is a single crystal with facets, which is similar to the results reported previously. 32,35

The top-view and cross-sectional SEM images of the asprepared nickel nanorod arrays on silicon substrates are displayed in Fig. 1. In the study of the morphology of nickel nanorods by SEM, the energy of the electron beam applied to the samples is $5 \mathrm{KeV}$ and the distance between the objective lens and the sample is about $7 \mathrm{~mm}$. The top-view images are arranged in the left column of Fig. 1, whereas the crosssectional images are in the right column. The SEM images in Figs. 1(a) and 1(b) represent the short nickel nanorods prepared by the glancing angle deposition in $30 \mathrm{~min}$ deposition time. The SEM nickel nanorods deposited in $50 \mathrm{~min}$ are shown in Figs. 1(c) and 1(d), and those deposited in $90 \mathrm{~min}$ are displayed in Figs. 1(e) and 1(f). A nickel thin film is deposited on a silicon substrate and serves as the reference. The representative SEM images of this reference sample are exhibited in Figs. 1(g) and 1(h). The cross-sectional images are taken from the freshly cleaved nickel samples. For the SEM images shown in Fig. 1, the samples are intentionally tilted by about $5-10^{\circ}$ from the vertical axis of the SEM column in order to expose the top surface of the samples. Sharp nanotips are clearly shown in the cross-sectional SEM images of the samples deposited in 50 and $90 \mathrm{~min}$. Some large nanorods in the sample deposited in $30 \mathrm{~min}$ also develop sharp nanotips at the top ends of the nanostructures. In terms of morphology, the nanorod sample grown in $30 \mathrm{~min}$ is similar to the nickel thin film deposited with normal incident deposition flux as shown in Figs. 1(g) and 1(h). In the SEM images of the nickel thin film, the characteristic columnar structure and long crack-like void boundaries between nickel nanograins can be seen, which are formed due to the limited surface diffusion and non-uniform flux in sputtering at normal incident angle. ${ }^{36}$

In this study, the nickel nanorod arrays with 90 min deposition time are annealed in forming gas in vacuum at 

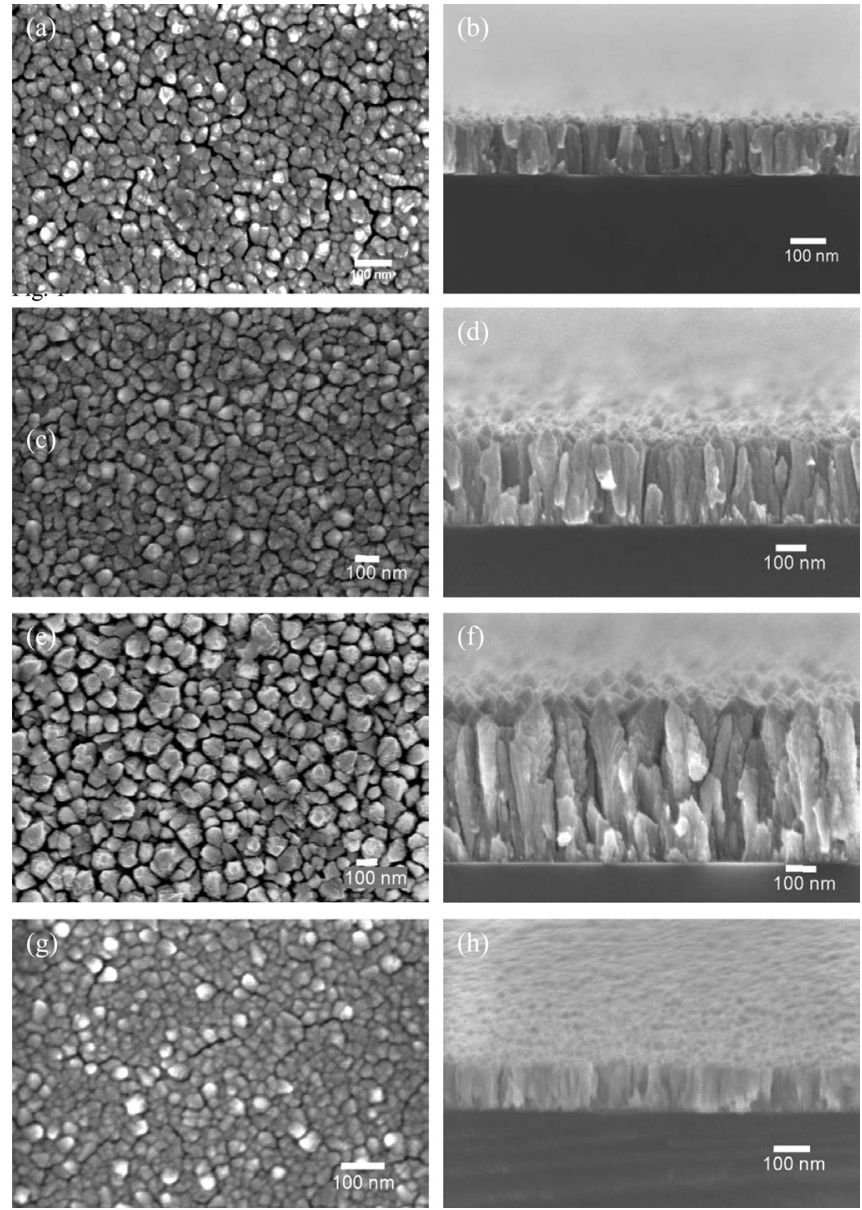

FIG. 1. SEM images of the as-prepared nickel nanorod arrays and a nickel thin film on silicon substrates. The top-view SEM images are arranged in the left column and the cross-sectional SEM images are shown in the right column. (a) and (b) are the SEM images of nanorods deposited in $30 \mathrm{~min}$; (c) and (d) are the SEM images of nanorods deposited in $50 \mathrm{~min}$; (e) and (f) are those deposited in $90 \mathrm{~min}$; and ( $\mathrm{g}$ ) and (h) are the SEM images of a nickel thin film, which serves as the reference. The white scale bars represent $100 \mathrm{~nm}$.

different temperatures: $300,400,500$, and $600{ }^{\circ} \mathrm{C}$. The annealing duration is $30 \mathrm{~min}$. The top-view SEM images of the samples after annealing are shown in Fig. 2. At $300^{\circ} \mathrm{C}$, there is no significant morphological change in the SEM images, as depicted in Fig. 2(a). When the temperature is increased to $400{ }^{\circ} \mathrm{C}$, the nanorods start to change some of their features. The edges of the rods are rounded up and some of the nanorods appear to merge together, creating small domains of nanorods. Accordingly, the crack-like voids are widened as can be seen in Fig. 2(b). The sharp nanotips become blunt at this annealing temperature. Nevertheless, every column still can be distinguished from the top-view and cross-sectional images. At 500 and $600{ }^{\circ} \mathrm{C}$, most of the individual nanorods are fused with their closest neighbors and form large domain structures, as demonstrated in Figs. 2(c) and 2(d). The domains are divided by wide irregular void boundaries. Small pores are also visible and form interconnected channels inside each domain. This porous surface pattern is similar to the so-called "mudcracking" pattern found in the columnar yttria-stabilized zirconia (YSZ) thin films after sintering at high temperatures. $^{37,38}$ It is found by modeling that the surface diffusion
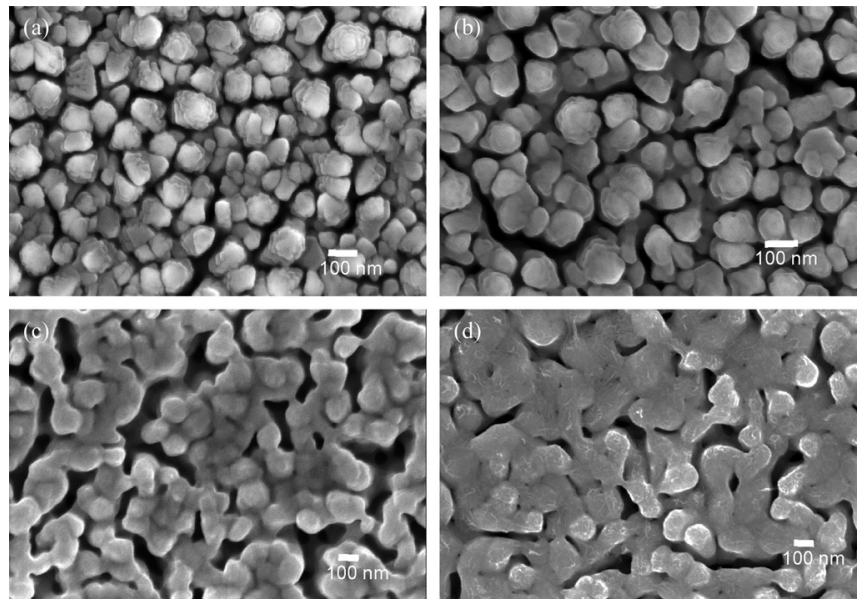

FIG. 2. SEM top-view images of nickel nanorod arrays after annealed at (a) $300^{\circ} \mathrm{C}$, (b) $400{ }^{\circ} \mathrm{C}$, (c) $500^{\circ} \mathrm{C}$, and (d) $600{ }^{\circ} \mathrm{C}$ for $30 \mathrm{~min}$. The nanorods are deposited by the glancing angle deposition technique in $90 \mathrm{~min}$ at $85^{\circ}$ deposition angle.

driven neck formation and coalescence are responsible for the mud-cracking pattern formation in YSZ thin films. ${ }^{37}$ In our experiment, the similar mud-cracking pattern observed in our samples also can be explained by the neck formation and merging during annealing process at a temperature above $500{ }^{\circ} \mathrm{C}$. The porous film obtained at $600{ }^{\circ} \mathrm{C}$ is denser than the one at $500^{\circ} \mathrm{C}$, while the surface appears smoother at higher annealing temperature due to surface diffusion.

From above results, it is clear that significant morphological change happens at $500^{\circ} \mathrm{C}$ annealing temperature. We studied the annealing effect of nanorods with different heights at $500^{\circ} \mathrm{C}$ for $30 \mathrm{~min}$ in forming gas. The mud-crack like pattern is also observed in all of the nickel nanorod and thin film samples after annealing at $500^{\circ} \mathrm{C}$ for $30 \mathrm{~min}$, as shown in Fig. 3. From the top-view images exhibited in the left column in Fig. 3, the characteristic two-sized pores, i.e., the large inter-domain gaps and the small intra-domain pores, are observed in the samples after heat treatment. The average width of the inter-domain gaps increases with the increasing heights of nanorods, while the number density of the nanorods enclosed in the domains seems decreasing with the heights. As the nanorods grow in height, the diameter also increases. The nanorods are more separated with increasing deposition time. Therefore, the neck formation between neighboring nanorods becomes difficult for taller nanorods than shorter ones. From the cross-sectional images arranged in the right column in Fig. 3, the columnar microstructure still exists after $30 \mathrm{~min}$ annealing at $500^{\circ} \mathrm{C}$ for all the nanorod arrays. Saw-tooth like features can be observed on the surface of the annealed nanorod samples, whereas the top surface of the annealed thin film is fairly flat. Furthermore, at the interface near the silicon substrate, a very thin layer of continuous film is formed, which may be due to the coalescence of very short and small nanorods deposited near the surface of the substrate in the early stage of the deposition. These short and small nanorods can be found in the cross-sectional SEM images of the as-deposited nanorod samples as shown in Fig. 1. They are the results of shadowing effect during the nanorod growth in glancing angle deposition. 

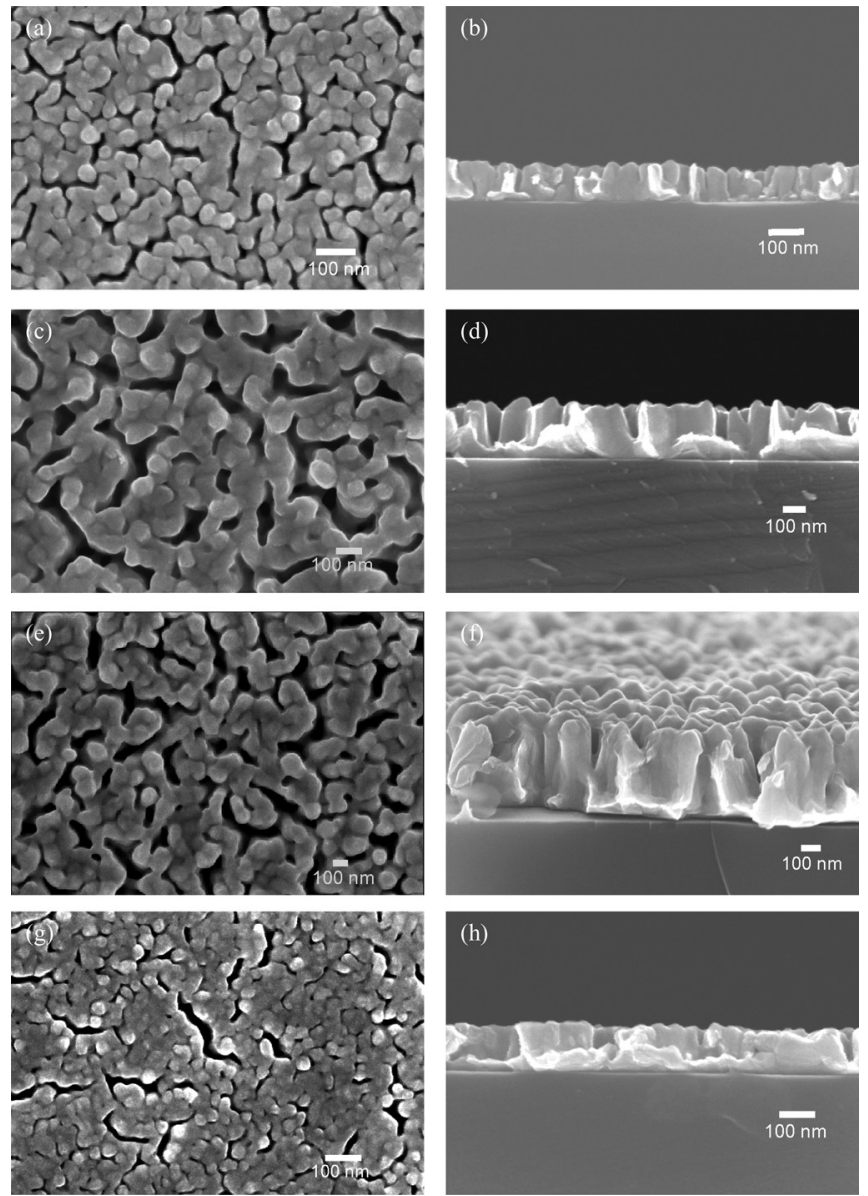

FIG. 3. SEM images of nickel nanorod arrays and thin film annealed at $500{ }^{\circ} \mathrm{C}$ for $30 \mathrm{~min}$. The top-view and cross-sectional images are arranged in the left and right columns, respectively. (a) and (b) are the SEM images of nanorod arrays deposited in $30 \mathrm{~min}$; (c) and (d) are the images of nanorod arrays deposited in $50 \mathrm{~min}$; (e) and (f) are the results of nanorod arrays deposited in $90 \mathrm{~min}$; and (g) and (h) are the results of the nickel thin film.

It can be observed that the average thickness of the nickel nanorod and thin film samples is reduced by the annealing process. The measured heights and thickness of the samples before and after annealing are listed in Table I. The nanorod sample prepared in 50 min deposition shrinks the most by $35.7 \%$ in height in all the samples, while the nanorod samples deposited in $90 \mathrm{~min}$ only reduce $5.5 \%$ of the average height of the nanorods. The shrinkage of the thin film is small as well, by about $10 \%$.

The as-deposited nanorods have large surface energy due to their high aspect ratios as demonstrated in Figs. 1(a)-1(f). The nanorods also have sharp apexes with nanoscaled radii. Thus, the surface diffusion is strong at elevated temperatures.

TABLE I. The measured average height of the nickel nanorods and the thickness of the nickel thin film before and after annealing at $500^{\circ} \mathrm{C}$ for 30 $\mathrm{min}$. The percentage of the reduction is calculated.

\begin{tabular}{lcccc}
\hline \hline & 30 minutes & 50 minutes & 90 minutes & Thin \\
& nanorod & nanorod & nanorod & film \\
\hline $\begin{array}{l}\text { Height before annealing }(\mathrm{nm}) \\
\begin{array}{l}\text { Height after annealing } \\
\text { at } 500^{\circ} \mathrm{C}(\mathrm{nm})\end{array}\end{array}$ & $135 \pm 5$ & $350 \pm 10$ & $550 \pm 10$ & $133 \pm 5$ \\
\begin{tabular}{l} 
Percentage of height reduction \\
\hline \hline
\end{tabular} & $25.9 \%$ & $35.7 \%$ & $5.5 \%$ & $9.8 \%$ \\
\hline
\end{tabular}

The apexes are suppressed after annealing at $500{ }^{\circ} \mathrm{C}$ and the diameters of the nanorods are increased due to the mass transport through the surface diffusion. As such, surface diffusion is the driving force of the shrinkage of nanorods in height, $d H / d t$, as expressed by the Nichols-Mullins' law, $d H / d t=A_{0} B / R^{3}$, where $A_{0}$ is a constant related to the geometry of the nanorods, $B$ is a material based constant at a specific temperature, and $R$ is the principal radius of the nanorods at the apex position. ${ }^{39}$ Constant $B$ is governed by $B=D_{s} \gamma \Omega^{4 / 3} / k T$, where $D_{s}$ is the surface diffusion coefficient, $\gamma$ is the surface energy, $\Omega$ is the atomic volume of the material, $k T$ is the thermal energy at the temperature $T .{ }^{39}$ Time dependent evolution of the nanorod morphology can be numerically simulated using the Nichols-Mullins' law, which is not the focus of this report and will be explored in the future. Nevertheless, we develop a simple geometric model to relate the change of porosity $p$ to the change of nanorod shapes. We assume the nanorods are cylinders with a radius $r$ and a height $H$. The cylinders are arranged in a triangular lattice with a center to center separation $S$. The porosity $p$ is thus determined by $p=1-(2 \pi / \sqrt{3})(r / S)^{2}$. It is necessary to point out that the porosity does not depend on the height $H$. In our annealing study, the radius $r$ of the nanorods is increasing with time while the separation $S$ remains a constant before the nanorods merge together. Therefore, the decreasing of porosity is expected from this simple model.

The crystallographic change in the nickel nanorod arrays after thermal treatment at $500^{\circ} \mathrm{C}$ is studied by the $\theta-2 \theta$ $\mathrm{X}$-ray diffraction (XRD) analysis. The XRD pattern of the nanorod arrays deposited in 90 min on a natively oxidized silicon substrate is taken from the as-deposited and annealed samples, as shown in Fig. 4. The XRD diffractogram covering from 43 to $57^{\circ}$ of the $2 \theta$ angles shows both the diffracted peaks due to the silicon substrate and the nickel nanorods, as well as the trace amount of nickel silicide at the interface. The silicon (311) at $\sim 56.4^{\circ}$ and silicon (220) at $\sim 47.9^{\circ}$ are the non-Bragg scattering peaks from the silicon (100) substrate we used in the experiments. ${ }^{40}$ The as-deposited nickel nanorod arrays demonstrate the inevitable formation of nickel silicide on the surface of the substrate in sputtering

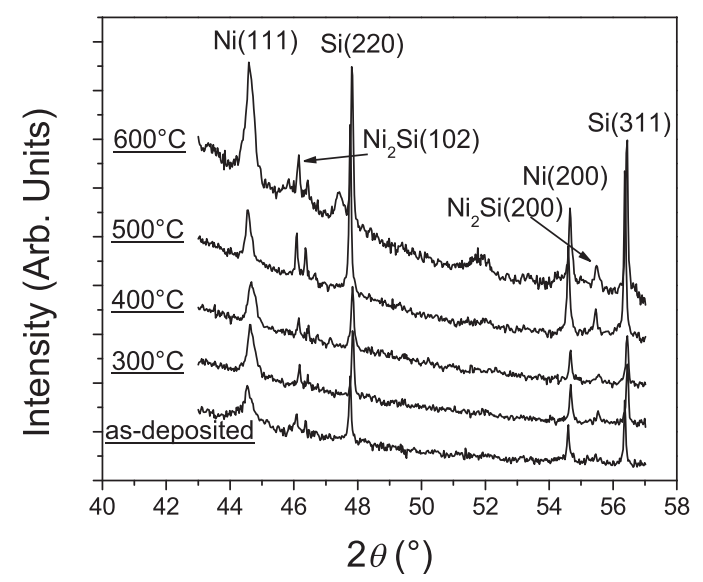

FIG. 4. XRD patterns of nickel nanorod arrays deposited in 90 min before and after annealing at $300,400,500$, and $600^{\circ} \mathrm{C}$. The intensity of nickel (111) and (200) peaks increases after annealing, indicating the growth of grains in the samples. 
deposition, mainly due to the energetic deposition flux. ${ }^{41}$ The intensity and the sharpness of the nickel silicide peaks increase with the annealing temperature varying from 300 to $600^{\circ} \mathrm{C}$, while the peaks remain as the minor ones in the XRD spectra. The intensity and the sharpness of the major nickel peaks in the diffraction pattern shown in Fig. 4 are enhanced after thermal treatment, which indicates the recrystallization and growth of the nickel crystals accompanying the morphological change.

The as-deposited nickel nanorods have a broad and weak peak of nickel (111) plane located at $2 \theta=44.7 \pm 0.1^{\circ}$, while the peak of nickel (200) located at $2 \theta=54.6 \pm 0.1^{\circ}$ is stronger and narrower than nickel (111) peak in the samples deposited in 50 and $90 \mathrm{~min}$. The nickel (200) peak is absent in the nanorod arrays prepared in $30 \mathrm{~min}$, as shown as the dashed line in the lower part of Fig. 5. The comparison of the XRD pattern before and after annealing for the nanorod samples deposited in 30 and $90 \mathrm{~min}$, respectively, is presented in Fig. 5. After annealing at $500^{\circ} \mathrm{C}$ for $30 \mathrm{~min}$, the intensity is increased significantly for both peaks of nickel (111) and nickel (200) for all samples, as can be seen from the XRD crystallogram in Fig. 5. The nickel XRD crystallographic peaks in all samples are analyzed in terms of the crystallite size $D$ by using the Debye-Scherrer equation $D=k \lambda /(W \cos \theta)$, where $k$ is a constant depends on the particle shape and varies between 0.89 and $1.39 \mathrm{rad}^{42} \lambda$ is the wavelength of the incident $\mathrm{X}$-ray $(\lambda=1.542 \AA), W$ is the full width at half maximum of the diffraction peak, and $\theta$ is the half Bragg angle at the center of the peak. In our calculation, we use $k=0.9$ as suggested by others. ${ }^{16,42}$ Table II summarizes the calculated the crystalline grain size $D$ of both nickel (111) and (200) peaks for all the samples before and after annealing. It is observed from the Table II that the grain size is proportional to the sample thickness. Moreover, it is noted that the grain size is increased in all samples after annealing. For instant, for nickel (111) peak, the diameter grows up after annealing by $105 \%$ in nickel nanorods with 30 min deposition, while only by about $26 \%$ in the 90 min sample. On the other hand, for nickel (200) peak, the grain size increases after annealing by $74 \%$ in $50 \mathrm{~min}$ deposition time, $41.5 \%$ in 90 min deposition time, and $28 \%$ in the nickel thin film.

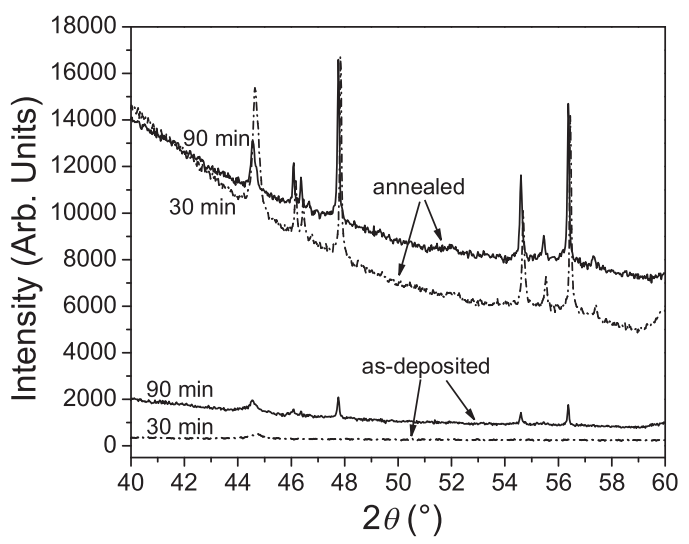

FIG. 5. Selected XRD diffractogram of nickel nanorod arrays deposited in $30 \mathrm{~min}$ (dashed lines) and $90 \mathrm{~min}$ (solid lines) before and after annealing at $500^{\circ} \mathrm{C}$ for $30 \mathrm{~min}$. The intensity of nickel diffracted peaks increases dramatically after annealing.
TABLE II. Average grain size $D$ in nickel nanorod and thin film samples calculated from the (111) and (200) XRD peaks by using the DebyeScherrer equation.

\begin{tabular}{lccc}
\hline \hline & & $\begin{array}{c}\text { Average } \\
\text { grain size D in } \\
(111)(\mathrm{nm})\end{array}$ & $\begin{array}{c}\text { Average } \\
\text { grain size D } \\
\text { in (200) (nm) }\end{array}$ \\
\hline Nanorods (30 min deposition) & As-deposited & $17 \pm 2$ & N/A \\
Nanorods (50 min deposition) & Annealed & $35 \pm 2$ & $92 \pm 5$ \\
& As-deposited & $25 \pm 2$ & $54 \pm 3$ \\
Nanorods (90 min deposition) & Annealed & $40 \pm 1$ & $94 \pm 4$ \\
Nickel thin film & Annealed & $38 \pm 1$ & $82 \pm 5$ \\
& As-deposited & $24 \pm 3$ & $116 \pm 6$ \\
& Annealed & $39 \pm 2$ & $87 \pm 5$ \\
\hline \hline
\end{tabular}

\section{CONCLUSION}

In conclusion, we have demonstrated experimentally the evolution of nickel nanorod arrays after heat treatment. The nanorods are deformed and connected to form a mud-crack like porous structure after annealing at $500{ }^{\circ} \mathrm{C}$. The pores are interconnected due to surface diffusion. Our XRD analysis reveals that the grain size in the porous mud-crack structure is increased after annealing. The intensity and sharpness of the diffracted nickel (111) and (200) peaks are enhanced by the thermal treatment, indicating the diffusion driven recrystallization of the nanorods. If the samples are annealed for long enough time, they are expected to be solidified completely. A continuous solid thin film should be formed at the end. However, the formation of nickel silicide may not be neglected under such situation. Practically, porous metal membranes are interesting for the applications in catalysis, fuel cell, lithium-ion battery, nanoelectronics, etc.

\section{ACKNOWLEDGMENTS}

E.A. is supported by the King Abdullah Scholarships program administrated by the Ministry of Higher Education, Saudi Arabia. Partial of this work is financially supported by the VCU Presidential Research Quest Fund. We thank Mr. Tyler Selden for valuable discussions.

${ }^{1}$ T. K. Sau, A. L. Rogach, F. Jackel, T. A. Klar, and J. Feldmann, Adv. Mater. 22, 1805 (2010).

${ }^{2}$ S. Eustis and M. A. El-Sayed, Chem. Soc. Rev. 35, 209 (2006).

${ }^{3}$ M. A. El-Sayed, Acc. Chem. Res. 34, 257 (2001).

${ }^{4}$ B. R. Cuenya, Thin Solid Films 518, 3127 (2010).

${ }^{5}$ S. Linic, P. Christopher, H. Xin, and A. Marimuthu, Acc. Chem. Res. 46, 1890 (2013).

${ }^{6}$ J. D. Aiken and R. G. Finke, J. Mol. Catal. A 145, 1 (1999).

${ }^{7}$ M. Auffan, J. Rose, J. Y. Bottero, G. V. Lowry, J. P. Jolivet, and M. R. Wiesner, Nat. Nanotechnol. 4, 634 (2009).

${ }^{8}$ T. Chookajorn, H. A. Murdoch, and C. A. Schuh, Science 337, 951 (2012).

${ }^{9}$ N. Combe, P. Jensen, and A. Pimpinelli, Phys. Rev. Lett. 85, 110 (2000).

${ }^{10}$ C. M. Tollan, R. Marcilla, J. A. Pomposo, J. Rodriguez, J. Aizpurua, J. Molina, and D. Mecerreyes, ACS Appl. Mater. Interfaces 1, 348 (2009).

${ }^{11}$ H. Petrova, J. Perez Juste, I. Pastoriza-Santos, G. V. Hartland, L. M. Liz-Marzan, and P. Mulvaney, Phys. Chem. Chem. Phys. 8, 814 (2006).

${ }^{12}$ S. Karim, M. E. Toimil-Molares, A. G. Balogh, W. Ensinger, T. W. Cornelius, E. U. Khan, and R. Neumann, Nanotechnology 17, 5954 (2006).

${ }^{13}$ H. Li, J. M. Biser, J. T. Perkins, S. Dutta, R. P. Vinci, and H. M. Chan, J. Appl. Phys. 103, 024315 (2008). 
${ }^{14}$ M. K. Santala and A. M. Glaeser, Acta Mater. 56, 1967 (2008).

${ }^{15}$ M. E. Toimil Molares, A. G. Balogh, T. W. Cornelius, R. Neumann, and C. Trautmann, Appl. Phys. Lett. 85, 5337 (2004).

${ }^{16}$ P. I. Wang, T. C. Parker, T. Karabacak, G. C. Wang, and T. M. Lu, Nanotechnology 20, 085605 (2009).

${ }^{17}$ T. Karabacak, J. S. DeLuca, P.-I. Wang, G. A. Ten Eyck, D. Ye, G.-C. Wang, and T.-M. Lu, J. Appl. Phys. 99, 064304 (2006).

${ }^{18}$ Z. F. Zhou, Y. C. Zhou, Y. Pan, and C. F. Xu, Acta Mater. 58, 3059 (2010).

${ }^{19}$ S.-S. Chang, C.-W. Shih, C.-D. Chen, W.-C. Lai, and C. R. C. Wang, Langmuir 15, 701 (1999).

${ }^{20}$ S. Karim, M. E. Toimil-Molares, W. Ensinger, A. G. Balogh, T. W. Cornelius, E. U. Khan, and R. Neumann, J. Phys. D: Appl. Phys. 40, 3767 (2007).

${ }^{21}$ L. Liu, W. Lee, R. Scholz, E. Pippel, and U. Gosele, Angew. Chem. 47, 7004 (2008).

${ }^{22}$ M. Rauber, F. Muench, M. E. Toimil-Molares, and W. Ensinger, Nanotechnology 23, 475710 (2012).

${ }^{23}$ C. Q. Sun, Y. Wang, B. K. Tay, S. Li, H. Huang, and Y. B. Zhang, J. Phys. Chem. B 106, 10701 (2002).

${ }^{24}$ Q. S. Mei and K. Lu, Prog. Mater. Sci. 52, 1175 (2007).

${ }^{25}$ Y. Chen, S. Milenkovic, and A. Hassel, Appl. Surf. Sci. 258, 6224 (2012).

${ }^{26}$ M. Dippel, A. Maier, V. Gimple, H. Wider, W. Evenson, R. Rasera, and G. Schatz, Phys. Rev. Lett. 87, 095505 (2001).
${ }^{27}$ P. Du and H. Wong, J. Appl. Phys. 111, 113503 (2012).

${ }^{28}$ S. P. A. Gill, Appl. Phys. Lett. 102, 143108 (2013).

${ }^{29}$ F. A. Nichols, J. Mater. Sci. 11, 1077 (1976).

${ }^{30}$ D. Ye, S. Mutisya, and M. Bertino, Appl. Phys. Lett. 99, 081909 (2011).

${ }^{31}$ K. Robbie and M. J. Brett, J. Vac. Sci. Technol. A 15, 1460 (1997).

${ }^{32}$ D. Ye, S. Moussa, J. D. Ferguson, A. A. Baski, and M. S. El-Shall, Nano Lett. 12, 1265 (2012).

${ }^{33}$ D. Vick, L. J. Friedrich, S. K. Dew, M. J. Brett, K. Robbie, M. Seto, and T. Smy, Thin Solid Films 339, 88 (1999).

${ }^{34}$ T. Karabacak, G. C. Wang, and T. M. Lu, J. Vac. Sci. Technol. A 22, 1778 (2004)

${ }^{35}$ D. X. Ye, T. M. Lu, and T. Karabacak, Phys. Rev. Lett. 100, 256102 (2008).

${ }^{36}$ B. Geetha Priyadarshini, S. Aich, and M. Chakraborty, J. Mater. Sci. 46, 2860 (2011)

${ }^{37}$ R. Krishnamurthy and D. J. Srolovitz, Acta Mater. 57, 1035 (2009).

${ }^{38}$ V. Lughi, V. K. Tolpygo, and D. R. Clarke, Mater. Sci. Eng., A 368, 212 (2004).

${ }^{39}$ F. A. Nichols and W. W. Mullins, J. Appl. Phys. 36, 1826 (1965).

${ }^{40}$ L. K. Bekessy, N. A. Raftery, and S. Russell, Adv. X-ray Anal. 50, 5 (2007).

${ }^{41}$ J. Kim, C.-S. Han, Y. C. Park, and W. A. Anderson, Appl. Phys. Lett. 92, 043501 (2008).

${ }^{42}$ A. Patterson, Phys. Rev. 56, 978 (1939). 